\title{
Synthesis of 1,2,3,4-Tetrahydroisoquinoline-2-sulfonic Acids
}

\author{
Ji Sun Lee, Sun Hee Kim, Han Sik Yoon, ${ }^{\dagger}$ and Chai-Ho Lee \\ Deparment of Chemistry, Wonkwang Cniversity, Jeonbuk 570-749. Korea \\ -School of Medical Radiation, Honkwang Helth Science College. Jeonbuk 570-750, Korea \\ Received Mav 7, 2003
}

Key Words : Tetrahydroisoquinoline. Sulfamic acid. Iminium ion

The 1,2.3,4-tetrahydroisoquinoline (THIQ) alkaloids 1. which are widely distributed in plant and animal kingdons. have received much attention because of their important biological activities. ${ }^{3}$ For example, 1-aryl- or 1-alkyl-1.2,3. 4-tetrahydroisoquinolines present in manmulian brain play a major role in therapy of variety of neurological disorders.<smiles>[R]c1cc2c(cc1[R])C([R])NCC2</smiles><smiles>[R]c1ccc(CCN)cc1[R]</smiles><smiles>[R]c1cc2c(cc1[R])C([R])[N]CC2</smiles>

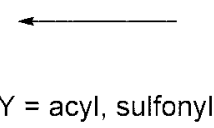<smiles>[R]c1ccc(CCN)cc1[R]</smiles>

3

Two general. acid mediated procedures have been reported for the preparation of THIQ ring sy stem. The first entails the Pictet-Spengler reaction of a 2-arylethylamine 2 with aldehyde. ${ }^{3}$ The second method requires the treatment of a $N$ substituted-2-ary lethylanime 3 (i.e. $N$-acyl ${ }^{+}$and $N$-sulfonyl group ${ }^{5}$ on the nitrogen) with carbonyl compound (i.e.. aldehyde. and the corresponding acetal). In these processes. introduction of an electron withdrawing substituents on nitrogen is to increase the electrophilicity of ininium intermediate. Previously. we demonstrated the intramolecularand intermolecular $\alpha$-sulfanidoalkylation transformation proceeding through the intermediacy of an ininium ion provide an expeditious route for the preparation of cyclic sulfamides containing THIQ ring. ${ }^{\circ}$ The sulfanic acid also have a sulfonic acid group as an electron withdrawing group on the nitrogen atom and is readily hydrolyzed to the

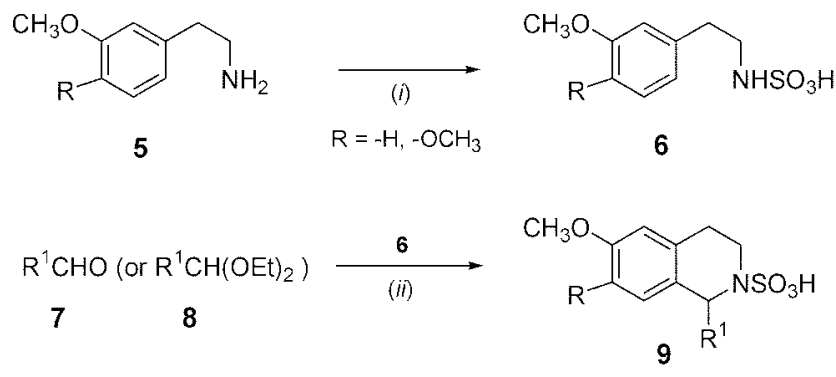

Scheme 1. Synthesis of 8 . Reagents and coditions: (i) 1 ) $\mathrm{ClSO}_{3} \mathrm{H}$ $\left.\mathrm{Et}_{3} \mathrm{~N},-5-0^{\circ} \mathrm{C}, 2\right) c-\mathrm{HCl} ;$ (ii) $\mathrm{HCO}_{2} \mathrm{H}$

corresponding amines. ${ }^{7}$

In this present study, we report on the reaction of $\mathrm{N}$-(2arylethyl)sulfonic acids 6 with aldehydes 7 (or the corresponding acetals 8 ) in formic acid for generation of $1.2,3.4$ tetralyydroisoquinoline-2-sulfonic acids 9 .

The starting sulfamic acid 6 were prepared according to established synthetic protocols. ${ }^{8}$ When amine $\mathbf{5}$ was reacted with $\mathrm{ClSO}_{3} \mathrm{H}-\mathrm{Et}_{3} \mathrm{~N}$ in chloroform. followed by treatment with hydrochloric acid, sulfamic acid 6 was formed in $60-70$ $\%$ yield. Intramolecular cyclization of 6 with 7 or $8 \mathrm{in}$ fomic acid $\left(96 \%\right.$ in $\mathrm{H}_{2} \mathrm{O}$ ) gave the desired product 9 in good yield Identification of all the isolated products 9 was accomplished with the aid of infrared. ${ }^{1} \mathrm{H}$ and ${ }^{13} \mathrm{C}$ NMR. and mass

Table 1. Reaction condition, mp, and yield of product 9

\begin{tabular}{|c|c|c|c|c|c|c|c|}
\hline \multirow{2}{*}{ Entry } & \multirow{2}{*}{ No. } & \multirow{2}{*}{$\mathrm{R}^{\mathrm{l}}$} & \multirow{2}{*}{$\mathrm{R}$} & \multicolumn{2}{|c|}{$\begin{array}{l}\text { Reaction } \\
\text { conditions }\end{array}$} & \multirow{2}{*}{$\operatorname{mp}_{\left({ }^{\circ} \mathrm{C}\right)^{\sigma}}$} & \multirow{2}{*}{$\begin{array}{l}\text { Yield } \\
(\%)^{b}\end{array}$} \\
\hline & & & & $\begin{array}{l}\text { Temp. } \\
\left({ }^{\circ} \mathrm{C}\right)\end{array}$ & $\begin{array}{l}\text { Time } \\
\text { (hi) }\end{array}$ & & \\
\hline l & $9 \mathbf{a}$ & $\mathrm{H}$ & $\mathrm{H}$ & It & l & $200-213$ & 81 \\
\hline 2 & $9 b$ & $\mathrm{H}$ & OMe & It & l & $172-200$ & 65 \\
\hline 3 & $9 c$ & $\mathrm{CH}_{2} \mathrm{Cl}$ & $\mathrm{H}$ & It & 12 & $75-80$ & 74 \\
\hline 4 & $9 d$ & $\mathrm{CH}_{2} \mathrm{Cl}$ & $\mathrm{OMe}$ & $\mathrm{rt}$ & 0.5 & $174-176$ & 92 \\
\hline 5 & $9 e$ & $\mathrm{CH}_{2} \mathrm{CN}$ & $\mathrm{H}$ & $\mathrm{rt}$ & 24 & $205-215$ & 65 \\
\hline 6 & $9 f$ & $\mathrm{CH}_{2} \mathrm{CN}$ & $\mathrm{OMe}$ & $\mathrm{rt}$ & 1 & $194-195$ & 68 \\
\hline 7 & $9 \mathrm{~g}$ & COOEt & $\mathrm{H}$ & It & 48 & $104-108$ & 58 \\
\hline 8 & $9 h$ & COOEt & $\mathrm{OMe}$ & $\mathrm{rt}$ & 48 & $170-171$ & 67 \\
\hline 9 & $9 \mathrm{i}$ & Benzyl & $\mathrm{H}$ & It & 24 & $142-145$ & 84 \\
\hline 10 & $9 \mathbf{j}$ & Benzyl & $\mathrm{OMe}$ & rt & 48 & $150-152$ & 73 \\
\hline 11 & $9 k$ & Phenyl & $\mathrm{H}$ & 50 & 24 & $169-171$ & 65 \\
\hline 12 & 91 & Phenyl & $\mathrm{OMe}$ & 50 & 24 & $172-176$ & 78 \\
\hline 13 & $9 m$ & $\begin{array}{l}\text { 3-methosy-4- } \\
\text { hydroxyphenyl }\end{array}$ & $\mathrm{H}$ & 50 & 24 & $117-119$ & 62 \\
\hline 14 & $9 n$ & $\begin{array}{l}\text { 3-methosy-4- } \\
\text { hydroxyphenyl }\end{array}$ & $\mathrm{OMe}$ & 50 & 24 & $76-99$ & 71 \\
\hline 15 & 90 & $\begin{array}{c}\text { 3,4,5-trimethoxy- } \\
\text { phenyl }\end{array}$ & $\mathrm{H}$ & It & 48 & $159-160$ & 66 \\
\hline 16 & $9 p$ & $\begin{array}{c}\text { 3,4,5-trimethoxy- } \\
\text { phenyl }\end{array}$ & OMe & 50 & 24 & $169-176$ & 68 \\
\hline 17 & $9 q$ & 2-furỵl & $\mathrm{H}$ & $\mathrm{rt}$ & 48 & $180-188$ & 70 \\
\hline 18 & $9 \mathbf{r}$ & 2-furryl & $\mathrm{OMe}$ & $\mathrm{rt}$ & 48 & $134-140$ & 74 \\
\hline 19 & $9 s$ & 2-thiophenyl & $\mathrm{H}$ & $\mathrm{rt}$ & 24 & $186-190$ & 70 \\
\hline 20 & $9 \mathrm{t}$ & 2-thiophenyl & $\mathrm{OMe}$ & $\mathrm{rt}$ & 24 & $230-238$ & 76 \\
\hline
\end{tabular}

Isolated yields. "Melting points are uncorrected. 
spectroscopy. In the infrared spectrum, the compound 9 exhibited characteristic absorption bands at 1246-1277 and $1016-1119 \mathrm{~cm}^{-1}$ for the sulfonyl group. Diagnostic signals of compounds 9 were observed at $4.38-6.13 \mathrm{ppm}$ in the ${ }^{\mathrm{H}} \mathrm{H}$ NMR spectra and at $48.5-60.8 \mathrm{ppm}$ in the ${ }^{13} \mathrm{C}$ NMR spectra for the methine (C-1) unit furnished by aldehdes 7 (or acetals 8).

In conclusion, we have developed a general and versatile method for the synthesis of 1. 2.3, 4-tetrahydroisoquinoline2 -sulfonic acids, by the reaction of $N$-(2-arylethyl)sulfamic acids with aldehydes (or acetals).

\section{Experimental Section}

Typical experimental procedure for synthesis of $N$ arylethylsulfamic acid 6 . A solution of arylethylamine 5 (10 mumol) and triethy lamine ( $12 \mathrm{mmol}$ ) in $30 \mathrm{~mL}$ of $\mathrm{CHCl}_{3}$ was stirred at $-5-0^{\circ} \mathrm{C}$ and chlorosulfonic acid ( $10 \mathrm{mmol}$ ) was added dropwise so as to maintain the temperature below 0 ${ }^{\circ} \mathrm{C}$. The solution was acidified with $\mathrm{I} N \mathrm{HCl}$ solution to $\mathrm{pH}$ 2 . The solid that precipitated was filtered to give the desired products 6.

2-(3-Methoxyphenyl)ethylsulfamic acid (6a): Beginning with 3-methoxyphenethylamine $(1.51 \mathrm{~g})$. compound $6 \mathrm{a}$ was obtained in $55 \%$ yield $\left(1.27 \mathrm{~g}\right.$ ): $\mathrm{mp} 1+4-146{ }^{\circ} \mathrm{C}$; IR (KBr) $3180(\mathrm{NH}) .1288,1063 \mathrm{~cm}^{-1} ;{ }^{1} \mathrm{H}$ NMR (DMSO-d 6$) \delta 2.87$ (t. $J=8.3 \mathrm{~Hz} .2 \mathrm{H}$ ), 3.21 (t. $J=8.3 \mathrm{~Hz}, 2 \mathrm{H}$ ). 3.75 (s. $3 \mathrm{H}$ ), 6.79-6.82 (m. 3H), 7.22-7.25 (m. lH). 10.18 (br s. IH) ppm; ${ }^{13} \mathrm{C}$ NMR (DMSO-d $\left.\mathrm{d}_{6}\right) \delta 32.5,45.6 .55 .5,112.9 .114 .7$. 121.3. 130.2, 139.4, $160.0 \mathrm{ppm}$ : LR FBA MS: calcd for $[\mathrm{M}-1]^{-} 230.1$. found 230.2 .

2-(3,4-Dimethoxyphenyl)ethylsulfamic acid (6b) Beginning with 3,4-dimethoxyphen-etlylamine $(1.81 \mathrm{~g})$. compound $6 \mathrm{~b}$ was obtained in $67 \%$ yield $(1.75 \mathrm{~g})$ : $\mathrm{mp} 164$ $170{ }^{\circ} \mathrm{C}$; IR (KBr) $3146(\mathrm{NH}), 1259.1072 \mathrm{~cm}^{-1}$; ${ }^{1} \mathrm{H}$ NMR (DMSO-d (D) $_{6} \delta 2.82(\mathrm{t}, J=8.3 \mathrm{~Hz} .2 \mathrm{H}) .3 .19(\mathrm{t}, J=8.3 \mathrm{~Hz}$. $2 \mathrm{H}), 3.71$ (s. $3 \mathrm{H}) .3 .75$ (s, $3 \mathrm{H}), 6.73$ (dd. $J=1.8 \mathrm{~Hz}$ and $J=$ $7.8 \mathrm{~Hz} .1 \mathrm{H}) .6 .82(\mathrm{~d} . J=1.8 \mathrm{~Hz} .1 \mathrm{H}) .6 .87(\mathrm{~d} J=7.8 \mathrm{~Hz}$. lH) 10.18 (br s. $1 \mathrm{H})$ ppm: ${ }^{13} \mathrm{C}$ NMR (DMSO-d $) \delta 32.1$. 45.9. 56.0. 56.1. 112.6. 113.0, 121.0,130.2, 184.2, 149.4 ppm: LR FBA MS: calcd for $[M-1]^{-} 260.1$. found 260.2 .

Typical experimental procedure for synthesis of $1,2,3$, 4-tetrahydroisoquinoline-2-sulfonic acid 9. A formic acid $\left(96 \%\right.$ in $\mathrm{H}_{2} \mathrm{O} .20 \mathrm{~mL}$ ) solution of arylethylsulfamic acids 6 (2.0 mmol) and aldehydes 7 (or acetals 8 ) (2.0 mmol) was stirred. and then the solution was quenched with $1 \mathrm{~N} \mathrm{HCl}(50$ $\mathrm{mL}$ ). The solid that precipitated was filtered and then recrystallized from methanol-chloroform to give the desired products 9 (Table 1 ).

9a: IR (KBr) 1269.1063 $\mathrm{cm}^{-1}$. 'H NMR (acetone-d 6 ) $\delta$ 3.13 (t. $J=6.4 \mathrm{~Hz}, 2 \mathrm{H}$ ). 3.59 (t. $J=6.4 \mathrm{~Hz}, 2 \mathrm{H}$ ). 3.73 (s. $3 \mathrm{H}) .4 .39$ (s. $2 \mathrm{H}) .6 .75(\mathrm{~d} . J=2.3 \mathrm{~Hz} . \mathrm{lH}) .6 .78(\mathrm{dd} . J=2.3$ $\mathrm{Hz}$ and $J=8.4 \mathrm{~Hz} .1 \mathrm{H}) .7 .12(\mathrm{~d} . J=8.4 \mathrm{~Hz} .1 \mathrm{H}) \mathrm{ppm}:{ }^{13} \mathrm{C}$ NMR (acetone-d 6 ) $\delta 26.3 .45 .9,48.5,54.9113 .1,113.4$. 120.8. 128.1 133.1. $159.3 \mathrm{ppm}$. LR FBA MS: calcd for [M-1] ${ }^{-} 242.1$. found 242.7

9b: IR (KBr) 1258.1057 $\mathrm{cm}^{-1}:{ }^{1} \mathrm{H}$ NMR (acetone-d $\left.\mathrm{d}_{6}\right) \delta$ $3.07(\mathrm{t}, J=6.4 \mathrm{~Hz} .2 \mathrm{H}) .3 .59(\mathrm{t} . J=6.4 \mathrm{~Hz} .2 \mathrm{H}), 3.74(\mathrm{~s}$, $6 \mathrm{H}) .4 .38$ (s. $2 \mathrm{H}) .6 .77$ (s. $1 \mathrm{H}), 6.79(\mathrm{~s}, \mathrm{lH}) \mathrm{ppm}:{ }^{13} \mathrm{C}$ NMR (acetone-d 6 ) $\delta 25.6 .46 .1,48.5 .55 .4 .55 .5,109.8$. 111.5. 120.5. 123.8, 148.2. $148.7 \mathrm{ppm}$ : LR FBA MS: calcd for [M-1] $]^{-} 272.1$. found 272.7 .

9c: IR (KBr) 1265. $1047 \mathrm{~cm}^{-1} ;{ }^{1} \mathrm{H}$ NMR (acetone-d $\left.\mathrm{d}_{6}\right) \delta$ 3.05 (ddd, $J=5.5 \mathrm{~Hz} . J=5.5 \mathrm{~Hz}$. and $J=17.6 \mathrm{~Hz}, 1 \mathrm{H}$ ). 3.18 (ddd, $J=5.5 \mathrm{~Hz} . J=8.3 \mathrm{~Hz}$. and $J=17.6 \mathrm{~Hz} . \mathrm{lH}$ ). 3.45 (ddd, $J=5.5 \mathrm{~Hz} . J=8.3 \mathrm{~Hz}$. and $J=13.2 \mathrm{~Hz} . \mathrm{lH}$ ), 3.69 (ddd $J=5.5 \mathrm{~Hz}, J=5.5 \mathrm{~Hz}$. and $J=13.2 \mathrm{~Hz} . \mathrm{lH}$ ). 3.74 (s. $3 \mathrm{H}$ ). 4.18 (dd. $J=7.1 \mathrm{~Hz}$ and $J=12.8 \mathrm{~Hz} . \mathrm{lH}$ ). 4.29 (dd. $J=$ $3.4 \mathrm{~Hz}$ and $J=12.8 \mathrm{~Hz}, 1 \mathrm{H}) .4 .94(\mathrm{dd}, J=3.4 \mathrm{~Hz}$ and $J=7.1$ Hz. $\mathrm{lH}), 6.79(\mathrm{~d}, J=2.7 \mathrm{~Hz}, 1 \mathrm{H}) .6 .83(\mathrm{dd}, J=2.7 \mathrm{~Hz}$ and $J$ $=8.7 \mathrm{~Hz}, 1 \mathrm{H}) .7 .30(\mathrm{~d}, J=8.7 \mathrm{~Hz}, \mathrm{lH}) \mathrm{ppm}:{ }^{13} \mathrm{C}$ NMR (acetone-d 6 ) $\delta 25.3 .40 .0,44.6 .55 .1 .55 .7,113.7 .113 .8$. 120.7. 127.9, 134.5. 159.5 ppm: LR FBA MS: calcd for [M-1] 290.0 . found 290.2

9d: IR (KBr) 1273, $1071 \mathrm{~cm}^{-1}:{ }^{1} \mathrm{H}$ NMR (acetone-d 6 3.03 (ddd, $J=5.5 \mathrm{~Hz} . J=5.8 \mathrm{~Hz}$. and $J=17.1 \mathrm{~Hz}, \mathrm{lH}$ ). 3.14 (ddd, $J=5.5 \mathrm{~Hz} . J=7.6 \mathrm{~Hz}$. and $J=17.1 \mathrm{~Hz}, 1 \mathrm{H}$ ), 3.50 (ddd, $J=5.8 \mathrm{~Hz} . J=7.6 \mathrm{~Hz}$. and $J=12.5 \mathrm{~Hz} . \mathrm{lH}), 3.74$ (ddd, $J=5.5 \mathrm{~Hz}, J=5.5 \mathrm{~Hz}$, and $J=12.5 \mathrm{~Hz} . \mathrm{lH}$ ). $3.78(\mathrm{~s}$, $6 \mathrm{H}) .4 .25(\mathrm{dd} . J=7.3 \mathrm{~Hz}$ and $J=12.9 \mathrm{~Hz}, \mathrm{lH}) .4 .38(\mathrm{dd} . J=$ $3.6 \mathrm{~Hz}$ and $J=12.9 \mathrm{~Hz}, 1 \mathrm{H}) .4 .97(\mathrm{dd}, J=3.6 \mathrm{~Hz}$ and $J=7.3$ $\mathrm{Hz}, \mathrm{lH}), 6.83$ (s. IH). 7.01 (s. lH) ppm: ${ }^{13} \mathrm{C}$ NMR (acetoned ) $\delta 24.6 .40 .1,44.7,55.3 .55 .6,55.8,109.7,112.0,120.4$. 125.3, 148.6. $149.5 \mathrm{ppm}$; LR FBA MS: calcd for $[\mathrm{M}-1]^{-}$ 320.0 . found 320.2

9e: IR (KBr) 1251. $1049 \mathrm{~cm}^{-1}$; ${ }^{1} \mathrm{H}$ NMR (methanol-d 3 ) $\delta$ 3.10 (ddd. $J=6.4 \mathrm{~Hz} . J=6.4 \mathrm{~Hz}$. and $J=17.5 \mathrm{~Hz}, 1 \mathrm{H}$ ). 3.18 (ddd, $J=6.4 \mathrm{~Hz}, J=7.1 \mathrm{~Hz}$, and $J=17.5 \mathrm{~Hz}, 1 \mathrm{H}$ ). 3.36 (dd, $J=5.0 \mathrm{~Hz}$ and $J=17.6 \mathrm{~Hz} . \mathrm{lH}$ ). 3.40 (dd. $J=6.1 \mathrm{~Hz}$ and $J=$ $17.6 \mathrm{~Hz} . \mathrm{lH}), 3.48(\mathrm{ddd}, J=6.4 \mathrm{~Hz} . J=6.4 \mathrm{~Hz}$, and $J=13.0$ $\mathrm{Hz}, 1 \mathrm{H}$ ), 3.64 (ddd. $J=5.5 \mathrm{~Hz}, J=7.1 \mathrm{~Hz}$, and $J=13.0 \mathrm{~Hz}$, lH). $3.80(\mathrm{~s}, 3 \mathrm{H}) .4 .89(\mathrm{dd}, J=5.0 \mathrm{~Hz}$ and $J=6.1 \mathrm{~Hz} . \mathrm{lH})$, 6.84 (d. $J=2.3 \mathrm{~Hz} . \mathrm{lH}$ ). 6.91 (dd. $J=2.3 \mathrm{~Hz}$ and $J=8.7 \mathrm{~Hz}$, lH). $7.3 \mathrm{~L}(\mathrm{~d} . J=8.7 \mathrm{~Hz}, \mathrm{lH}) \mathrm{ppm} ;{ }^{13} \mathrm{C}$ NMR (methanol-d 3 ) $\delta 22.0,24.9,39.6 .51 .1 .54 .6 .113 .5 .113 .8,115.8,121.1$. 127.6. 133.3. $160.2 \mathrm{ppm}$ : LR FBA MS: calcd for $[\mathrm{M}-1]^{-}$ 281.1. found 281.7 .

9f: IR (KBr) 1271, $1071 \mathrm{~cm}^{-1}:{ }^{1} \mathrm{H}$ NMR (acetone-d 6 ) $\delta$ $3.05-3.09$ (m. 2H). 3.38 (dd, $J=5.0 \mathrm{~Hz}$ and $J=17.9 \mathrm{~Hz}$. $1 \mathrm{H}$ ). 3.54 (dd. $J=5.5 \mathrm{~Hz}$ and $J=17.9 \mathrm{~Hz}, 1 \mathrm{H}$ ), 3.54 (ddd. $J$ $=5.0 \mathrm{~Hz}, J=5.7 \mathrm{~Hz}$. and $J=12.7 \mathrm{~Hz} \cdot 1 \mathrm{H}$ ). 3.69 (ddd $J=$ $5.5 \mathrm{~Hz}, J=7.3 \mathrm{~Hz}$ and $J=12.7 \mathrm{~Hz}, 1 \mathrm{H}$ ). $3.76(\mathrm{s.} 3 \mathrm{H}) .3 .77$ (s. $3 \mathrm{H}$ ). 4.97 (dd. $J=5.0 \mathrm{~Hz}$ and $J=5.5 \mathrm{~Hz}, 1 \mathrm{H}$ ). 6.82 (s. 1H). $6.96(\mathrm{~s} .1 \mathrm{H}) \mathrm{ppm}:{ }^{13} \mathrm{C}$ NMR (acetone-d 6 ) $\delta 22.7,24.3$. 39.5, 50.6. 55.4. 55.6. 109.5, 111.9. 116.8. 120.9, 124.7. 148.4 , 149.5 ppm: LR FBA MS: calcd for [M-1] ${ }^{-} 311.1$. found 311.7 .

9g: IR ( $\mathrm{KBr}) 1248.1016 \mathrm{~cm}^{-1}:{ }^{1} \mathrm{H}$ NMR (acetone-d $\left.\mathrm{d}_{6}\right) \delta$ 1.29 (t. $J=7.1 \mathrm{~Hz}, 3 \mathrm{H}$ ) 3.14 (ddd $J=5.5 \mathrm{~Hz}, J=5.7 \mathrm{~Hz}$. and $J=17.2 \mathrm{~Hz}, \mathrm{lH}$ ). 3.24 (ddd $J=5.5 \mathrm{~Hz} . J=8.7 \mathrm{~Hz}$, and $J=17.2 \mathrm{~Hz}, 1 \mathrm{H}$ ). 3.67 (ddd $J=5.5 \mathrm{~Hz} . J=5.7 \mathrm{~Hz}$, and $J=$ $12.3 \mathrm{~Hz}, 1 \mathrm{H}$ ). $3.77-3.81$ (m. $1 \mathrm{H}) .3 .80$ (s. $3 \mathrm{H}) .4 .31$ (q. $J=$ $7.1 \mathrm{~Hz} .2 \mathrm{H}), 5.45$ (s. $1 \mathrm{H}) .6 .85$ (d. $J=2.7 \mathrm{~Hz} .1 \mathrm{H}) .6 .89$ (dd. $J=2.7 \mathrm{~Hz}$ and $J=8.7 \mathrm{~Hz}, 1 \mathrm{H}), 7.46(\mathrm{~d}, J=8.7 \mathrm{~Hz}, 1 \mathrm{H})$ 
ppm: ${ }^{13} \mathrm{C}$ NMR (acetone-d $\mathrm{d}_{6}$ ) $\delta$ 13.4. 25.0. 39.9, 54.9. 55.7, 62.8, 113.4. 113.7. 117.9, 129.1. 134.1. 160.0, 167.6 ppm: LR FBA MS: calcd for [M-1] ${ }^{-}$315.1. found 315.7 .

9h: IR (KBr) 1273. $1061 \mathrm{~cm}^{-1}:{ }^{1} \mathrm{H}$ NMR $\left(\mathrm{CDCl}_{3}\right) \delta 1.33$ (t. $J=7.3 \mathrm{~Hz}, 3 \mathrm{H}$ ) 2.96 (ddd. $J=3.2 \mathrm{~Hz}, J=3.2 \mathrm{~Hz}$, and $J=$ $16.5 \mathrm{~Hz} .1 \mathrm{H}$ ), 3.14 (ddd. $J=4.1 \mathrm{~Hz} . J=12.4 \mathrm{~Hz}$. and $J=$ $16.5 \mathrm{~Hz} .1 \mathrm{H}$ ), 3.45 (ddd. $J=3.2 \mathrm{~Hz} . J=12.4 \mathrm{~Hz}$. and $J=$ $12.4 \mathrm{~Hz} . \mathrm{lH}), 4.21$ (ddd, $J=3.2 \mathrm{~Hz}, J=3.2 \mathrm{~Hz}$. and $J=12.4$ $\mathrm{Hz}, 1 \mathrm{H}), 3.86(\mathrm{~s}, 3 \mathrm{H}), 3.87(\mathrm{~s}, 3 \mathrm{H}), 4.35$ (q. $J=7.3 \mathrm{~Hz} .2 \mathrm{H})$. 5.43 (s. 1H), 6.65 (s. 1H), 6.93 (s, lH) ppm: ${ }^{13} \mathrm{C}$ NMR $\left(\mathrm{CDCl}_{3}\right) \delta$ 14.1. 26.2. 45.9, 56.1. 56.2, 60.8, 64.3. 109.7. 111.2. 124.8. 148.8, 149.8. 149.7, 169.0 ppm: LR FBA MS: calcd for $[\mathrm{M}-1]^{-} 344.1$. found 344.7

9i: IR (KBr) 1246, $1059 \mathrm{~cm}^{-1}:{ }^{1} \mathrm{H}$ NMR (methanol-d $\left.\mathrm{d}_{\hat{j}}\right) \delta$ 3.07 (ddd, $J=6.0 \mathrm{~Hz}, J=6.9 \mathrm{~Hz}$. and $J=16.3 \mathrm{~Hz}, 1 \mathrm{H}$ ). 3.12 (dd. $J=8.2 \mathrm{~Hz}$ and $J=13.9 \mathrm{~Hz}, 1 \mathrm{H}$ ), 3.16 (ddd. $J=6.0 \mathrm{~Hz}$. $J=7.1 \mathrm{~Hz}$. and $J=16.3 \mathrm{~Hz}, \mathrm{IH}$ ), 3.33 (ddd. $J=6.0 \mathrm{~Hz} . J=$ $7.1 \mathrm{~Hz}$. and $J=12.6 \mathrm{~Hz} . \mathrm{LH}) .3 .49$ (dd, $J=6.4 \mathrm{~Hz}$ and $J=$ $13.8 \mathrm{~Hz}, 1 \mathrm{H}$ ), 3.53 (ddd, $J=6.0 \mathrm{~Hz} . J=6.9 \mathrm{~Hz}$. and $J=12.6$ $\mathrm{Hz}, 1 \mathrm{H}), 3.78(\mathrm{~s}, 3 \mathrm{H}), 4.77$ (dd. $J=6.4 \mathrm{~Hz}$ and $J=8.2 \mathrm{~Hz}$. $1 \mathrm{H}), 6.79(\mathrm{~d} . J=2.3 \mathrm{~Hz}, 1 \mathrm{H}$ ). $6.8 \mathrm{l}$ (dd. $J=2.3 \mathrm{~Hz}$ and $J=$ $8.2 \mathrm{~Hz} . \mathrm{lH}$ ). 7.05 (d, $J=8.2 \mathrm{~Hz} . \mathrm{lH}) .7 .31-7.35$ (n. $3 \mathrm{H}$ ). 7.37-7.41 (m, 2H) ppm; ${ }^{13} \mathrm{C}$ NMR (methanol-d d $^{2} \delta 25.2$. $39.2,39.8,54.5,56.4,113.1 .113 .2,123.4 .127 .5,127.9$. 128.9. 129.4, 132.8, 135.3. 129.7 ppm; LR FBA MS: calcd for $[\mathrm{M}-1]^{-} 332.1$. found 332.7 .

9j: IR (KBr) 1262, $1061 \mathrm{~cm}^{-1} ;{ }^{1} \mathrm{H}$ NMR (methanol-d $\left.\mathrm{d}_{3}\right) \delta$ 3.04 (ddd, $J=3.9 \mathrm{~Hz}, J=6.2 \mathrm{~Hz}$, and $J=17.4 \mathrm{~Hz}, 1 \mathrm{H}$ ). 3.09 (ddd, $J=6.0 \mathrm{~Hz}, J=7.6 \mathrm{~Hz}$, and $J=17.4 \mathrm{~Hz} . \mathrm{IH}$ ). 3.18 (ddd, $J=3.9 \mathrm{~Hz}, J=7.6 \mathrm{~Hz}$, and $J=13.3 \mathrm{~Hz} .1 \mathrm{H}$ ). 3.36 (ddd, $J=6.0 \mathrm{~Hz} . J=6.2 \mathrm{~Hz}$, and $J=13.3 \mathrm{~Hz} . \mathrm{lH}$ ), 3.42 (dd. $J=7.3 \mathrm{~Hz}$ and $J=13.7 \mathrm{~Hz} .1 \mathrm{H}) .3 .55(\mathrm{dd}, J=7.4 \mathrm{~Hz}$ and $J=$ $13.7 \mathrm{~Hz}, 1 \mathrm{H}), 3.67$ (s. $3 \mathrm{H}), 3.81$ (s. $3 \mathrm{H}), 4.74$ (dd. $J=7.3 \mathrm{~Hz}$ and $J=7.3 \mathrm{~Hz} . \mathrm{HH}) .6 .42(\mathrm{~s} .1 \mathrm{H}), 6.81(\mathrm{~s} .1 \mathrm{H}), 7.31-7.35(\mathrm{~m}$, $3 \mathrm{H})$, 7.38-7.41 (m, 2H) ppm: ${ }^{13} \mathrm{C}$ NMR (nethanol-d $\left.\mathrm{d}_{3}\right) \delta$ $24.5,39.1 .40 .0,53.8$. 55. 1, 55.3. 110.3. 111.8. 123.2. 124.0. 127.4. 129.0, 130.0. 135.9, 147.6. 149.0 ppm: LR FBA MS: calcd for $[\mathrm{M}-1]^{-} 362.1$, found 362.7 .

9k: IR (KBr) 1269. $1067 \mathrm{~cm}^{-1}$ : ${ }^{1} \mathrm{H}$ NMR (acetone-d 6 ) $\delta$ 3.15 (ddd $J=5.5 \mathrm{~Hz}, J=5.5 \mathrm{~Hz}$, and $J=17.4 \mathrm{~Hz}, 1 \mathrm{H}$ ). 3.30 (ddd $J=6.5 \mathrm{~Hz}, J=8.7 \mathrm{~Hz}$, and $J=17.4 \mathrm{~Hz}, 1 \mathrm{H}$ ). 3.47 (ddd $J=5.5 \mathrm{~Hz}, J=8.7 \mathrm{~Hz}$ and $J=12.9 \mathrm{~Hz}, 1 \mathrm{H}$ ). 3.53 (ddd $J=5.5 \mathrm{~Hz}, J=6.5 \mathrm{~Hz}$ and $J=12.9 \mathrm{~Hz}, 1 \mathrm{H}$ ) 3.80 (s. $3 \mathrm{H}) .5 .66(\mathrm{~s} .1 \mathrm{H}) .6 .72(\mathrm{~d} . J=8.7 \mathrm{~Hz}, 1 \mathrm{H}), 6.78(\mathrm{dd}, J=2.8$ $\mathrm{Hz}$ and $J=8.7 \mathrm{~Hz}, 1 \mathrm{H}), 6.88(\mathrm{~d}, J=2.8 \mathrm{~Hz}, 1 \mathrm{H}), 7.35-7.37$ (m, 2H) 7.46-7.48 (m, 3H) ppm: ${ }^{13} \mathrm{C}$ NMR (acetone-d 6 ) $\delta$ $25.3,40.0,55.0,59.2,113.1,113.6,123.7,129.1,129.4$. 129.6. 130.1 134.2. 137.0. 159.3 ppm. LR FBA MS: calcd for $[\mathrm{M}-1]^{-} 318.1$, found 318.7 .

9l: IR (KBr) 1262. $1063 \mathrm{~cm}^{-1}$ : 'H NMR (methanol-d ${ }^{\text {) }} \delta$ 3.11 (ddd $J=5.5 \mathrm{~Hz}, J=6.0 \mathrm{~Hz}$ and $J=17.4 \mathrm{~Hz}, 1 \mathrm{H}$ ). 3.23 (ddd $J=6.0 \mathrm{~Hz}, J=6.4 \mathrm{~Hz}$, and $J=17.4 \mathrm{~Hz}, 1 \mathrm{H}$ ). 3.43 (ddd $J=5.5 \mathrm{~Hz}, J=7.3 \mathrm{~Hz}$ and $J=13.0 \mathrm{~Hz}, 1 \mathrm{H}$ ). 3.49 (ddd $J=6.0 \mathrm{~Hz}, J=6.4 \mathrm{~Hz}$ and $J=13.0 \mathrm{~Hz}, 1 \mathrm{H}$ ) .3 .59 (s. $3 \mathrm{H}$ ) 3.86 (s. 3H). 5.69 (s. 1H). 6.34 (s. 1H). 6.90 (s. 1H). $7.35-7.37(\mathrm{~m} .2 \mathrm{H}) .7 .47-7.49(\mathrm{~m}, 3 \mathrm{H}) \mathrm{ppm}:{ }^{13} \mathrm{C}$ NMR (methanol- $\left.\mathrm{d}_{3}\right) \delta 24.5,39.5,55.1,55.3,59.3,110.7,111.4$.
$122.9,124.8,129.1,129.7,129.8,136.4,148.3,149.5$ ppm; LR FBA MS: calcd for [M-1] ${ }^{-} 348.1$. found 348.7

9m: IR (KBr) 1263, $1034 \mathrm{~cm}^{-1} ;{ }^{1} \mathrm{H}$ NMR (methanol-d $\left.\mathrm{d}_{3}\right) \delta$ 3.12 (ddd, $J=5.5 \mathrm{~Hz} . J=5.5 \mathrm{~Hz}$. and $J=17.6 \mathrm{~Hz}, 1 \mathrm{H}$ ). 3.30 (ddd, $J=6.2 \mathrm{~Hz} . J=8.2 \mathrm{~Hz}$, and $J=17.6 \mathrm{~Hz}, \mathrm{lH}$ ), 3.46 (ddd, $J=5.5 \mathrm{~Hz} . J=8.2 \mathrm{~Hz}$. and $J=12.7 \mathrm{~Hz} . \mathrm{HH}$ ) 3.53 (ddd, $J=5.5 \mathrm{~Hz} . J=6.2 \mathrm{~Hz}$. and $J=12.7 \mathrm{~Hz} . \mathrm{HH}$ ). 3.83 (s. $3 \mathrm{H}$ ). 3.79 (s. $3 \mathrm{H}$ ). 5.60 (s. $1 \mathrm{H}$ ). 6.75 (d, $J=1.9 \mathrm{~Hz}$ and $J=$ $8.3 \mathrm{~Hz}, \mathrm{lH}$ ). 6.79 (s. $2 \mathrm{H}$ ), 6.84 (d, $J=8.3 \mathrm{~Hz} .2 \mathrm{H}$ ), 6.95 (d. $J$ $=1.9 \mathrm{~Hz} \mathrm{lH}$ ) ppm: ${ }^{13} \mathrm{C}$ NMR (methanol-d $\left.\mathrm{d}_{3}\right) \delta 25.2 .39 .8$. $54.5,55.2,59.7,112.7,112.8,113.5,115.3,122.7 .123 .6$. 127.5. 129.3. 133.5. 147.9. 148.2. 159.7 ppm LR FBA MS: calcd for $[\mathrm{M}-1]^{-} 364.1$, found 364.7 .

9n: IR (KBr) 1260, $1059 \mathrm{~cm}^{-1} ;{ }^{1} \mathrm{H}$ NMR (acetone-d 6 ) $\delta$ 3.08 (ddd, $J=6.0 \mathrm{~Hz} . J=6.0 \mathrm{~Hz}$. and $J=17.2 \mathrm{~Hz}, 1 \mathrm{H}$ ). 3.20 (ddd, $J=6.4 \mathrm{~Hz} . J=7.6 \mathrm{~Hz}$. and $J=17.2 \mathrm{~Hz} . \mathrm{lH}$ ), 3.42 (ddd, $J=6.0 \mathrm{~Hz} . J=7.6 \mathrm{~Hz}$. and $J=12.8 \mathrm{~Hz}$. $\mathrm{lH}$ ), 3.49 (ddd, $J=6.0 \mathrm{~Hz}, J=6.4 \mathrm{~Hz}$. and $J=12.8 \mathrm{~Hz}, 1 \mathrm{H}) .3 .62(\mathrm{~s}$, $3 \mathrm{H}) .3 .84$ (s. $3 \mathrm{H}$ ). 3.85 (s. $3 \mathrm{H}$ ). $5.6 \mathrm{l}$ (s. $1 \mathrm{H}) .6 .39$ (s, lH), $6.76(\mathrm{dd}, J=1.9 \mathrm{~Hz}$ and $J=8.0 \mathrm{~Hz} .1 \mathrm{H}), 6.85(\mathrm{~d}, J=8.0$ $\mathrm{Hz} .1 \mathrm{H}$ ). 6.87 (s, IH). 6.94 (d. $J=1.9 \mathrm{~Hz} .1 \mathrm{H}) \mathrm{ppm}:{ }^{13} \mathrm{C}$ NMR (acetone-d $\mathrm{d}_{6}$ ) $\delta 24.8 .4 .04 .55 .2 .55 .4 .55 .9 .59 .5,111.3$. 111.6. 113.5, 114.9, 123.2, 123.6. 125.2. 128.0, 147.1, 147.9. 148.4. $149.6 \mathrm{ppm}$; LR FBA MS: calcd for $[\mathrm{M}-1]^{-}$ 394 . 1 . found 394.7 .

90: IR (KBr) 1277. $1057 \mathrm{~cm}^{-1}$ : ${ }^{\text {H }} \mathrm{H}$ NR (acetone-d 6 ) $\delta$ 3.13 (ddd, $J=5.0 \mathrm{~Hz} . J=5.5 \mathrm{~Hz}$. and $J=17.5 \mathrm{~Hz}, 1 \mathrm{H}$ ). 3.41 (ddd, $J=6.0 \mathrm{~Hz} . J=9.9 \mathrm{~Hz}$. and $J=17.5 \mathrm{~Hz} .1 \mathrm{H}$ ), 3.55 (ddd. $J=5.5 \mathrm{~Hz} . J=9.9 \mathrm{~Hz}$. and $J=12.7 \mathrm{~Hz} . \mathrm{lH}$ ). 3.68 (ddd, $J=5.0 \mathrm{~Hz}, J=5.5 \mathrm{~Hz}$. and $J=12.7 \mathrm{~Hz}, \mathrm{lH}) .3 .71(\mathrm{~s}$, $3 \mathrm{H}$ ). 3.73 (s. $6 \mathrm{H}$ ). 3.80 (s. $3 \mathrm{H}$ ). 6.03 (s. $1 \mathrm{H}$ ). 6.62 (s, $2 \mathrm{H}$ ), $6.80(\mathrm{~d}, J=2.8 \mathrm{~Hz}$ and $J=8.7 \mathrm{~Hz}, 1 \mathrm{H}), 6.87(\mathrm{~d} . J=2.8 \mathrm{~Hz}$, $2 \mathrm{H}$ ). $6.96\left(\mathrm{~d}_{,} J=8.7 \mathrm{~Hz} \mathrm{lH}\right.$ ) ppm; ${ }^{13} \mathrm{C}$ NMR (acetone-d 6 ) $\delta$ 25.2, 40.6. 54.9, 55.9, 59.8. 59.9, 107.4, 113.1, 113.5. 123.6. $129.4,132.3 .134 .1,138.8 .153 .7,159.4$ ppm; LR FBA MS: calcd for $[\mathrm{M}-1]^{-} 408.1$, found 804.7 .

9p: IR (KBr) 1261,1051 cm cm $^{-1} \mathrm{H}$ NMR (acetone-d 6 ) $\delta$ 3.10 (ddd $J=5.5 \mathrm{~Hz} . J=5.7 \mathrm{~Hz}$, and $J=17.4 \mathrm{~Hz}, 1 \mathrm{H}$ ). 3.30 (ddd $J=6.4 \mathrm{~Hz} . J=8.0 \mathrm{~Hz}$, and $J=17.4 \mathrm{~Hz}, 1 \mathrm{H}$ ). 3.52 (ddd $J=5.5 \mathrm{~Hz}, J=8.0 \mathrm{~Hz}$, and $J=13.8 \mathrm{~Hz}, 1 \mathrm{H}$ ). 3.62 (ddd $J=5.7 \mathrm{~Hz}, J=6.4 \mathrm{~Hz}$, and $J=13.8 \mathrm{~Hz}, 1 \mathrm{H}$ ). 3.06 (s. $3 \mathrm{H}$ ). 3.72 (s. $3 \mathrm{H}$ ). 3.75 (s. $6 \mathrm{H}$ ). 3.81 (s. $3 \mathrm{H}$ ). 5.75 (s. $1 \mathrm{H}$ ). 6.43 (s. 1H), 6.74 (s. 2H), 6.87 (s. 1H) ppm: ${ }^{13} \mathrm{C}$ NMR (acetone-d6) $\delta 25.0,41.0,54.8,56.0 .56 .2,56.4 .60 .4 .60 .9$. 107.2. 110.6, 111.2, 122.5, 124.7, 131.5, 138.8, 148.2 . 149.3, 153.6 ppm. LR FBA MS: calcd for [M-1] $]^{-} 438.1$. found 438.7

9q: IR (KBr) $1246.1115 \mathrm{~cm}^{-1}:{ }^{\text {] }} \mathrm{H}$ NMR (methanol-d 3 ) $\delta$ 3.17 (t. $J=6.4 \mathrm{~Hz}, 2 \mathrm{H}), 3.42-3.52(\mathrm{~m}, 2 \mathrm{H}), 5.80$ (s. $1 \mathrm{H})$, $6.42(\mathrm{~d} . J=3.2 \mathrm{~Hz}, 1 \mathrm{H}), 6.49(\mathrm{dd} . J=1.8 \mathrm{~Hz}$ and $J=3.2 \mathrm{~Hz}$. $1 \mathrm{H}) .6 .83(\mathrm{dd}, J=2.3 \mathrm{~Hz}$ and $J=8.7 \mathrm{~Hz}, 1 \mathrm{H}), 6.86(\mathrm{~d}, J=$ $2.3 \mathrm{~Hz}, 1 \mathrm{H}), 6.95(\mathrm{~d}, J=8.7 \mathrm{~Hz} \mathrm{lH}), 7.63(\mathrm{~d}, J=1.8 \mathrm{~Hz}, 1 \mathrm{H})$ ppm: ${ }^{13} \mathrm{C}$ NMR (methanol-d $\left.\mathrm{d}_{3}\right) \delta 24.9 .39 .0 .52 .2 .54 .8$. $110.8,112.9,113.2,113.6,120.7,128.9 .133 .3,144.7$. 148.9. 159.8 ppm: LR FBA MS: calcd for [M-1]- 308.1 . found 308.7

9r: IR (KBr) $1265,1119 \mathrm{~cm}^{-1}$ : ${ }^{1} \mathrm{H}$ NMR (methanol-d 3 ) $\delta$ 
3.12 (t. $J=6.4$ Hz. $2 \mathrm{H}$ ), $3.4 \mathrm{l}-3.50$ (nt. $2 \mathrm{H}), 3.70(\mathrm{~s}, 3 \mathrm{H})$. 3.85 (s, 3H). $5.8 \mathrm{l}(\mathrm{s} .1 \mathrm{H}), 6.44(\mathrm{~d}, J=3.2 \mathrm{~Hz} .1 \mathrm{H}), 6.50$ (dd. $J=1.8 \mathrm{~Hz}$ and $J=3.2 \mathrm{~Hz} \mathrm{lH}), 6.57(\mathrm{~s} . \mathrm{lH}) .6 .87(\mathrm{~s}, \mathrm{lH})$. 7.65 (d. $J=1.8 \mathrm{~Hz}, 1 \mathrm{H}$ ) ppm: ${ }^{13} \mathrm{C}$ NMR (nethanol-d ${ }^{\text {) }} \delta$ $24.3,38.3,52.1,55.3,110.4 .110 .8,111.6 .113 .0,120.4$. 124.5. 144.7, 148.4, 148.9. 149.7 ppm; LR FBA MS: calcd for $[\mathrm{M}-1]^{-3} 38.1$, found 338.6

9s: IR (KBr) 1269. $1055 \mathrm{~cm}^{-1} ;{ }^{1} \mathrm{H}$ NMR (acetone-d $\left.\mathrm{d}_{6}\right) \delta$ 3.17 (ddd, $J=6.4 \mathrm{~Hz}, J=7.1 \mathrm{~Hz}$, and $J=17.5 \mathrm{~Hz}, \mathrm{IH}$ ), 3.23 (ddd, $J=5.9 \mathrm{~Hz}, J=6.4 \mathrm{~Hz}$, and $J=17.5 \mathrm{~Hz} .1 \mathrm{H}$ ). 3.48 (ddd, $J=6.4 \mathrm{~Hz}, J=6.4 \mathrm{~Hz}$, and $J=12.7 \mathrm{~Hz} .1 \mathrm{H}$ ). 3.54 (ddd, $J=5.9 \mathrm{~Hz}, J=7.1 \mathrm{~Hz}$, and $J=12.7 \mathrm{~Hz}, \mathrm{lH}$ ), 3.80 (s. $3 \mathrm{H}), 6.06(\mathrm{~s}, \mathrm{IH}), 6.82(\mathrm{dd}, J=2.3 \mathrm{~Hz}$ and $J=8.7 \mathrm{~Hz} . \mathrm{IH}$ ). $6.97(\mathrm{~d}, J=8.7 \mathrm{~Hz}, \mathrm{IH}) .7 .12(\mathrm{dd}, J=3.2 \mathrm{~Hz}$ and $J=5.4 \mathrm{~Hz}$. lH), 7.25 (d. $J=3.2 \mathrm{~Hz}, \mathrm{lH}$ ). 7.58 (dd. $J=0.9 \mathrm{~Hz}$ and $J=$ $5.4 \mathrm{~Hz}, \mathrm{IH}$ ) ppm: ${ }^{13} \mathrm{C}$ NMR (acetone-d 6 ) $\delta 25.1 .28 .9,53.5$. 55.0, 113.1. 113.6, 123.5. 127.7, 128.5. 129.6, 130.6. 133.6. 139.6. 159.5 ppm: LR FBA MS: calcd for $[\mathrm{M}-1]^{-} 324.0$. found $32+2$.

9t: IR (KBr) 1263, $1117 \mathrm{~cm}^{-1}$ : ${ }^{1} \mathrm{H}$ NMR (acetone-d $\left.\mathrm{d}_{6}\right) \delta$ 3.16 (t. $J=6.4$ Hz. $2 \mathrm{H}$ ), $3.47-3.56$ (nt. $2 \mathrm{H}), 3.79(\mathrm{~s}, 3 \mathrm{H})$. 3.80 (s. $3 \mathrm{H}) .6 .13$ (s. 1H), 6.60 (s, 1H), 6.85 (s. 1H). 7.07 (dd. $J=3.2 \mathrm{~Hz}$ and $J=5.0 \mathrm{~Hz}, \mathrm{IH}$ ). 7.35 (d. $J=3.2 \mathrm{~Hz} . \mathrm{IH}$ ). 7.53 (dd, $J=0.9 \mathrm{~Hz}$ and $J=5.0 \mathrm{~Hz}, \mathrm{IH}$ ) ppm: ${ }^{13} \mathrm{C}$ NMR (acetone-d $\mathrm{d}_{6}$ ) $\delta 24.3 .38 .5 .53 .2,55.3 .55 .4,111.2,111.5$. 123.1. 124.7. 127.7. 128.5, 130.7, 139.7, 148.2, 149.7 ppm; LR FBA MS: calcd for [M-1] 354 .1. found 354.1 .

Acknowledgements. This work was supported by grant No. R01-2001-000-00133-0 from the Korea Science \& Engineering Foundation.

\section{References}

1. (a) Padwa. A.: Beall. L. S.: Heidelbauugh. T. M.: Liu. B:
Sheehan, S. M. J. Org Chem. 2000, 65. 2684. (b) Glunewald, G L.: Dahanukar. V. H.: Teoh. B.: Criscione. K. R. J. Hed. Chent. 1999. 42. 1982. (c) Mitor. D. L.: Wyrick. S. D.: Charitsont. P. S.: Watts. V. J.: Nochols. D. E.: Mailman. R. B. J. Med. Chem. 1994. 37.4328 .

2. (a) Shinohara, T.: Toda, J.; Sano. T. Chem. Pham Bull. 1997. 45(5). 813. (b) Ornstein, P. L.: Anold. M. B.: Allen. N. K.: Bleisch. T.: Borromeo. P. S.: Lugar. C. W.: Leander. T. D.: Lodge. D.: Schoepp. D. D. J. Med Chem. 1996. 39. 2219. (c) Munchhof. M. T.: Meyers. A. I. J. Org Chem. 1996. 61. 4607. (d) Caritsonot. P. S. Bowen. J. P. Wyrick. S. D. Hoftman. A. J.; Cory. M. McPhail, A. T; Mailman, R. B. J. Hed. Chem. 1989, 32. 2650. (f) Clark. M. T.: Adejare. A. G.; Feller. D. R:; Miller, D. D. J. Med. Chent 1987. 30.86. (g) Battersby: A. R.: Southgate. R.: Staunton1. J.: Hurst. M J. Chent. Soc. (C) 1966. 1052.

3. (a) Eric. D.: Cook. I. M. Chem. Rev. 1995. 95(6). 1797. (b) Whaley, W. M.; Govindachari. T. R Org. React. 1951. 6. 151.

4. (a) Speckamp. W. N.: Moolenar. M. J. Tetrahedion 2000. 56. 3817. (b) Venkov. A. P.: Lukanov, L. K. Stmhesis 1989. 59. (c) Mollov. N. M.: Venkov. A. P. Sinthesis 1978. 62

5. (a) Barn. D. R.: Caulfield. W. L.: Cottney. T.: MaGurk. K.: Morphy. J. R.: Rankowic. Z.: Roberts. B. Bioorg. \& Med 2001.9. 2609. (b) Kohno, H.; Sekine. Y. Heterocycles 1996, 42(1). 14l. (c) Lukanov. L. K. Venkov, A. P.: Mollov, M. Synthesis 1987. 204.

6. (a) Lee. J. S.; Yang. I. D.: Kim. S. H. An. S. I.: Lee, C.-H. Bull. Korean Chem. Soc. 2003. 24(1). 129. (b) Lee. T. D.: Lee. C.-H.: Nakamura. H.: Ko. I.: Kang. S. O. Tetrohedon Lett. 2002. +3. 5483. (c) Lee. J. S.: Lee. C.-H. Bull. Korean Chem. Soc, 2002. 23(1). 167. (d) Lee. J. S.; Lee, C.H J. Korean Chem Soc. 2001. 45. 92. (e) Kong. Y. J.; Kim, S. H.: Lee, C.-H. J. Korean Chem. Soc. $1999,43,131$. (f) Lee. C.-H; Kohn. H. J. Hetenowclic Chent. 1990. 27. 2107. (g) Lee. C.-H.: Kohn. H. J. Ong Chem. 1990. 55. 6098. (h) Lee. C.-H.: Koht1. H. Heterocycte 1988. 27. 2581

7. (a) Kim. B. M.: So. S. M. Tetrahedron Lett. 1998. 39. 5381 . (b) Benson. G. A.: Spillane. W. J. Chem. Rev. 1980. 80, 151 .

8. (a) Shi. D.-F.: Bradshaw, T. D.: Chua, M.-S.: Westwell. A. D. Stevens. M. F. Bioorg. \& Hed. Chem. Leth. 2001, M, 1093. (b) Currant. W. V: Ross. A. A.: Lee. V. T. J. Antibiot. 1988. DZI(IO). 1418.

9. Nakanish. K: Solomon. P. H. Infrared Absoption Spectroscopy. Holden-Day: San Francisco. 1977. 